Orthopäde 2021 · 50:866-870

https://doi.org/10.1007/s00132-021-04140-6

Angenommen: 16. Juli 2021

Online publiziert: 17. August 2021

๑) Der/die Autor(en) 2021

\title{
Die ventrale Abstützung bei dorsalen lumbalen Fusionsoperationen
}

\section{Ein Fallbericht mit vielen Zwischenfällen}

Moritz Mederake - Christian Walter

Orthopädische Universitätsklinik Tübingen, Tübingen, Deutschland

\section{Zusammenfassung}

Die ventrale Abstützung im Rahmen dorsaler Fusionsoperationen ist insbesondere bei instabilen Pathologien, wie Spondylolisthesen oder Spondylodiszitiden, entscheidend für das Operationsergebnis. Der komplikative Verlauf einer Patientin mit simultan bestehender Spondylolisthese und Spondylodiszitis wird dargestellt und anhand der Literatur und des eigenen Behandlungsalgorithmus reevaluiert. Bei alleiniger Spondylodiszitis ist ein Beckenkamminterponat als Abstützung ausreichend. Bei zusätzlichen Störungen des sagittalen Profils ist eine Cage-Implantation zu bevorzugen.

\section{Schlüsselwörter}

Lumbalregion · Wirbelkörperfusion · Wirbelsäule · Spondylodiszitis · Spondylolisthese

\section{Anamnese}

Die 51-jährige Patientin klagte über ausgeprägte Lumbofemoralgien beidseits mit Hypästhesien an beiden ventralen Oberschenkeln. Zudem beeinträchtigte sie der massive Lotüberhang des Oberkörpers (- Abb. 1). Nebenbefundlich besteht eine chronische Hepatitis-C-Infektion und Methadon-Substitution bei Z.n. i.v.-Drogenabusus.
Als Ausgangssituation bestand 9 Jahre zuvor eine Spondylodiszitis bei Spondylolisthesis vera LWK5/SWK1 Meyerding Typ II, welche zunächst mit einer dorsalen Spondylodese und Bandscheibenresektion ohne ventrale Abstützung versorgt wurde (- Abb. 2).

Bei weiter bestehenden Infektzeichen waren weitere Revisionen, u.a. eine Beckenkammspongiosainterposition am 14. postoperativen Tag, erforderlich.

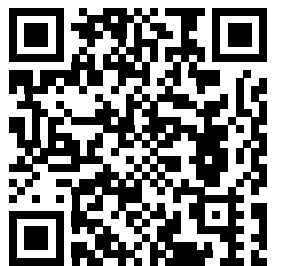

QR-Code scannen \& Beitrag online lesen
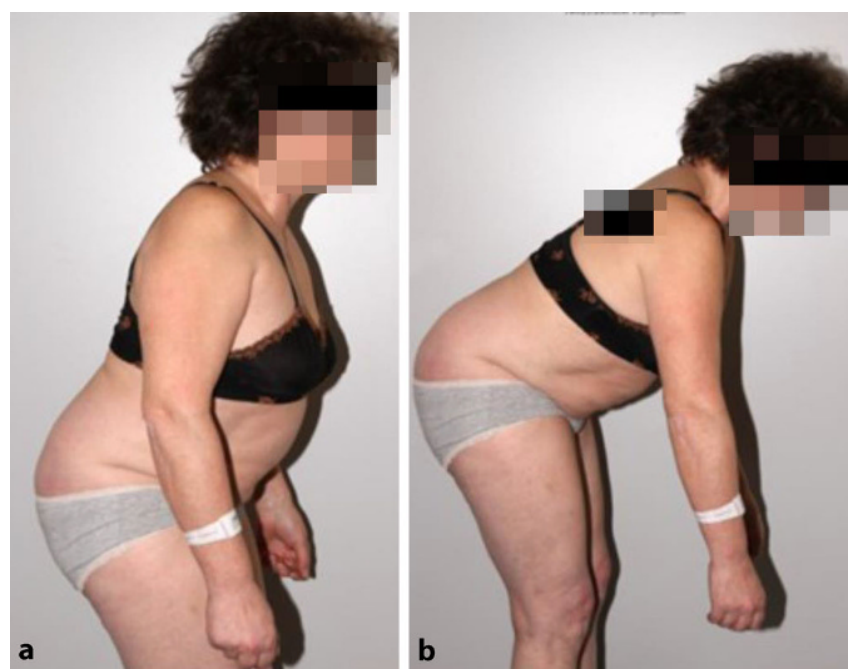

Abb. 1 Klinische Befund 9 Jahre nach Primäroperation (a maximale Reklination, $\mathbf{b}$ maximale Inklination) 

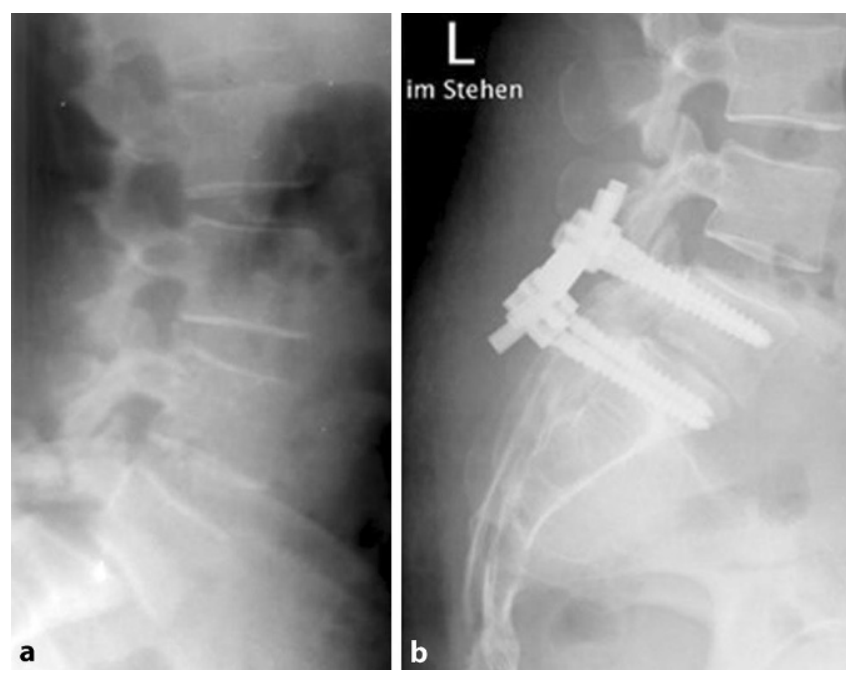

Abb. $2<$ Radiologischer Ausgangsbefund (a) und postoperativer Status nach Primäroperation (b)

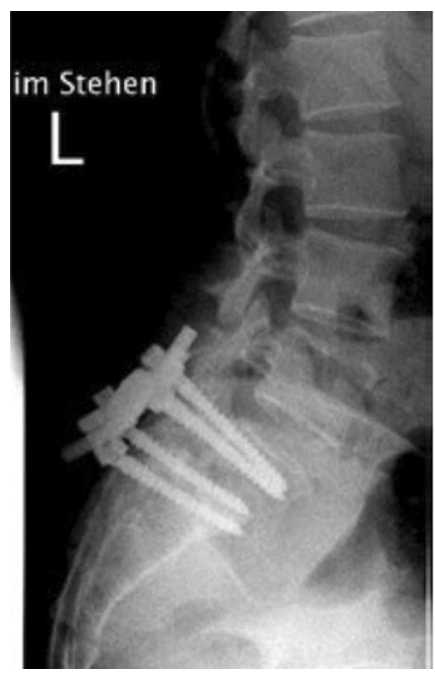

Abb. 3 A Verlaufskontrolle nach 3 Monaten: Eine Einschmelzung des Lendenwirbelkörpers 5 mit lokaler Kyphosierung von ca. $15^{\circ}$ stellt sich dar
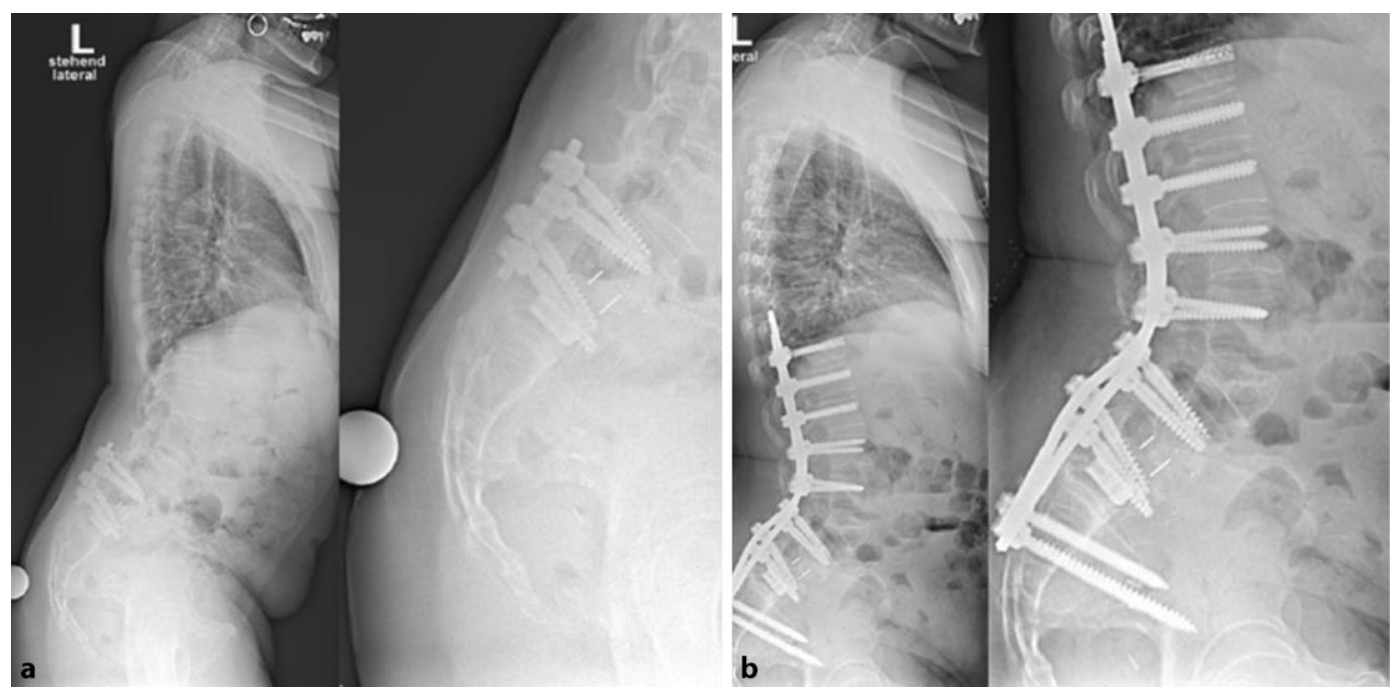

Abb. $4 \varangle$ Radiologischer Befund prä- (a) und postoperativ (b) nach Pedikelsubtraktionsosteotomie
Allerdings fand sich bereits nach einem Monat eine Schraubenlockerung mit progredienter Einschmelzung des LWK5 und lokaler Kyphosierung (• Abb. 3), welche

\section{Abkürzungen}

ALIF „Anterior lumbar interbody fusion”

BWK Brustwirbelkörper

LL Lumbale Lordose

LWK Lendenwirbelkörper

LWS Lendenwirbelsäule

PLIF "Posterior lumbar interbody fusion"

PSO Pedikelsubtraktionsosteotomie

PT Pelvic Tilt

SS Sacral Slope

SWK Sakralwirbelkörper

TLIF "Transforaminal lumbar interbody fusion" zu einer Verlängerung bis LWK4 mit ventraler trikortikaler Spantransplantation vom Beckenkamm 3 Monate nach der Primäroperation führte.

Trotz multipler weiterer Eingriffe (- Tab. 1) zeigte sich in den folgenden 5 Jahren eine sagittale Imbalance mit progredienter Lotabweichung nach ventral.

\section{Befund}

Radiologisch fanden sich hochpathologische Parameter für das sagittale Profil: So betrug die lumbale Lordose (LL) präoperativ nur $31^{\circ}$. Pelvic Tilt (PT) $37,1^{\circ}$ und Sacral Slope (SS) $47,9^{\circ}$ bei einer Pelvic In- cindence von $85^{\circ}$, waren ebenfalls außerhalb der Norm. Das C7-Lot stand deutlich vor den Hüftköpfen $(9,9 \mathrm{~cm}$ Abstand zur Hinterkante des SWK1).

\section{Diagnose}

Chronische, therapieresistente Lumbofemoralgie bei sagittaler Imbalance durch $30^{\circ}$ Defizit der LL bei Z.n. Fusion LWK4SWK1.

\section{Therapie und Verlauf}

Wir stellten die Indikation zur dorsalen Verlängerungsspondylodese von BWK10 bis Ilium mit 95 mm SWK2-Ala-Iliumschrau- 


\begin{tabular}{|c|c|c|}
\hline Zeitpunkt & Diagnose & Operation \\
\hline \multirow[t]{3}{*}{ Primäroperation } & \multirow{3}{*}{$\begin{array}{l}\text { Abszedierende Spondylodiszitis } \\
\text { LWK5/SWK1 bei Spondylolisthesis vera } \\
\text { LWK5/SWK1 Meyerding-Typ II }\end{array}$} & 1. Bandscheibenresektion und dorsale Spondylodese LWK5/SWK1 \\
\hline & & 2. Revision und Jet-Lavage 4 Tage nach Primäroperation \\
\hline & & $\begin{array}{l}\text { 3. Erneutes Debridement und Spongiosatransplantation von dorsal } \\
14 \text { Tage nach Primäroperation }\end{array}$ \\
\hline \multirow{2}{*}{$\begin{array}{l}3 \text { Monate nach Primäropera- } \\
\text { tion }\end{array}$} & \multirow{2}{*}{$\begin{array}{l}\text { Lockerung des Fixateur intern LWK5/SWK1 } \\
\text { mit Einschmelzung LWK5 }\end{array}$} & 4. Verlängerungsspondylodese LWK4-SWK1 mit Schraubenwechsel \\
\hline & & 5. Ventrale Spondylodese mit Beckenkammspan \\
\hline \multirow[t]{2}{*}{$\begin{array}{l}14 \text { Monate nach Primäropera- } \\
\text { tion }\end{array}$} & \multirow[t]{2}{*}{$\begin{array}{l}\text { Hypolordose und Fehlhaltung lumbosa- } \\
\text { kral mit Pseudarthrose LWK5/SWK1 sowie } \\
\text { ptotischem LWK5 versus SWK1 }\end{array}$} & $\begin{array}{l}\text { 6. Dorsoventrodorsale Spondylodese LWK4-SWK1 mit Re-Spondylode- } \\
\text { se dorsal, ventraler Cage-Implantation LWK5/SWK1 sowie Nachreposi- } \\
\text { tion dorsal LWK5/SWK1 }\end{array}$ \\
\hline & & 7. Interposition eines Cages LWK4/5 mit ventraler Aufrichtung \\
\hline 5 Jahre nach Primäroperation & $\begin{array}{l}\text { Implantatfehllage mit Pseudarthrose } \\
\text { LWK4/5 und in Kyphose konsolidierter } \\
\text { Olisthese LWK5/SWK1 }\end{array}$ & 8. Metallentfernung und Schraubenreinstrumentierung LWK4/5 \\
\hline
\end{tabular}

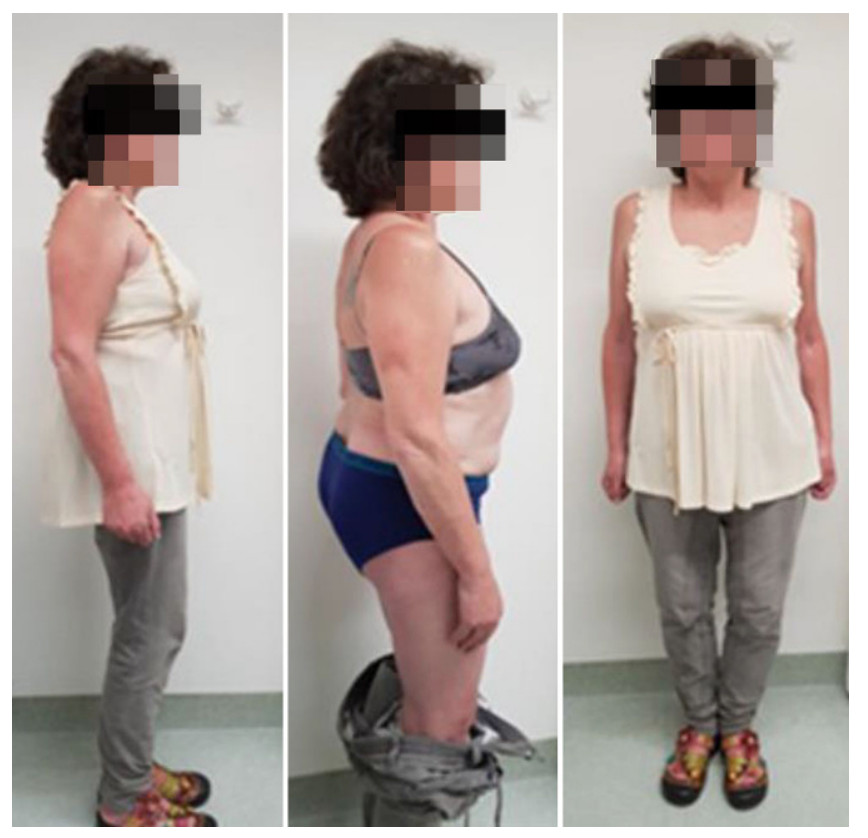

Abb. $5<$ Postoperativer klinischer $\mathrm{Be}$ fund ben und Pedikelsubtraktionsosteotomie (PSO) LWK3 und führten diese komplikationslos unter Neuromonitoring durch (- Abb. 4). Die Operationsdauer betrug $6 \mathrm{~h}$ mit einem Blutverlust von 1,5l. Trotz intraoperativ festgestellter ausgeprägter Narbenbildung im Bereich der voroperierten Gebiete ist diese Operationsdauer im Einklang mit der bestehenden Literatur [1]. Die LL verbesserte sich in der postoperativen Ganzwirbelsäulenaufnahme auf $60^{\circ}\left(+29^{\circ}\right)$. PT und SS veränderten sich weniger deutlich auf $33,8^{\circ}\left(-3,3^{\circ}\right)$ bzw. $51,2^{\circ}\left(+3,3^{\circ}\right)$. Das C7-Lot verbesserte sich ebenfalls deutlich auf einen Abstand von $3 \mathrm{~cm}$ zur Hinterkante des SWK1.
Im 3-Monats-Follow-up berichtet die Patientin über eine vollständige Regredienz der Lumbofemoralgien und über eine exzellente Korrektur des sagittalen Profils (• Abb. 5).

\section{Diskussion}

Der aufgezeigte komplikative Verlauf der Patientin, welcher in einer langstreckigen Korrekturspondylodese endete, wirft nun verschiedene Fragen auf.

Die Indikation zum primären operativen Eingriff wurde aufgrund der diagnostizierten Spondylodiszitis LWK5/SWK1 bei vorbestehender Spondylolisthese gestellt. Zu diskutieren ist, ob ein Zwischenwirbel- körperinterponat die Einschmelzung des LWK5 mit lokaler Kyphosierung und damit die multiplen Folgeoperationen hätte verhindern können. So konnten Polly et al. nachweisen, dass eine Cage-Implantation die Steifigkeit des Konstrukts signifikant erhöht [7]. Weiter entlastet der Cage die eingebrachten Schrauben, was einer Schraubenlockerung entgegenwirkt und die $L L$ verbessern kann $[2,5,6]$.

Verschiedene Studien zeigen hierbei exzellente Ergebnisse bei Implantation von Interponaten [3, 8], allerdings keinen Unterschied zwischen Cages unterschiedlicher Größe (TLIF-, ALIF- oder PLIF-Cages) [9].

Alternativ kann jedoch auch körpereigener Knochen verwendet werden: Ein trikortikaler Beckenkammspan ohne Reposition der Olisthese gilt in einigen skandinavischen Zentren weiter als Goldstandard und zeigt auch bei der Spondylodiszitis gute Ergebnisse [3, 4]. Aus heutiger Sicht wäre neben einer sorgfältigen Ausräumung des Bandscheibenfachs bereits bei der initialen Operation ein Interponat indiziert gewesen. In unserer Klinik implantieren wir primär einen Titan-Cage mit möglichst großem Fußabdruck.

Die in diesem Fall erforderliche PSO wurde gewählt, um durch die möglichst kaudale Korrektur die entstandene Kyphosierung zu kompensieren und die harmonische Lordosierung der LWS, soweit möglich, wiederherzustellen. Eine Osteotomie auf Höhe des LWK4 war aufgrund von Narbenbildungen nach den Voroperationen nicht möglich. Alternativ hätte auch ei- 
ne Cage-Implantation von LWK1 bis LWK4 diskutiert werden können. Allerdings wäre bei dieser Technik durch die Korrektur der lumbalen Lordose im kranialen Abschnitt der LWS laut Planung das C7-Lot weniger effektiv nach dorsal verlagert worden.

\section{Fazit für die Praxis}

- Im Sonderfall einer kombinierten Spondylodiszitis mit Spondylolisthese sollte, wenn möglich, für die Verwendung eines Cages mit großem Fußabdruck plädiert werden.

- Besteht eine Störung des sagittalen Profils, so ist diese zu adressieren und mittels einer Cage-Implantation oder einer Osteotomie zu versorgen. Dabei sollte die Indikation zu Osteotomien der vorderen Säule (wie z. B. Pedikelsubtraktionsosteotomie) zurückhaltend gestellt werden.

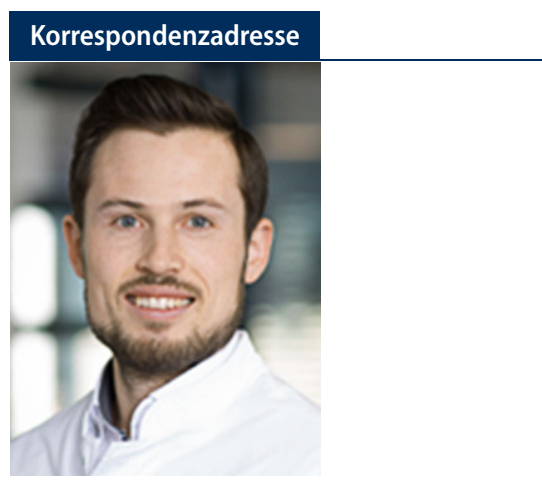

\section{Dr. Moritz Mederake, MD}

Orthopädische Universitätsklinik Tübingen Hoppe-Seyler-Str. 3, 72076 Tübingen, Deutschland

moritz.mederake@med.uni-tuebingen.de

Funding. Open Access funding enabled and organized by Projekt DEAL.

\section{Einhaltung ethischer Richtlinien}

Interessenkonflikt. M. Mederake und C. Walter geben an, dass kein Interessenkonflikt besteht.

Für diesen Beitrag wurden von den Autoren keine Studien an Menschen oder Tieren durchgeführt. Für die aufgeführten Studien gelten die jeweils dort angegebenen ethischen Richtlinien. Für Bildmaterial oder anderweitige Angaben innerhalb des Manuskripts, über die Patienten zu identifizieren sind, liegt von ihnen und/oder ihren gesetzlichen Vertretern eine schriftliche Einwilligung vor.

Open Access. Dieser Artikel wird unter der Creative Commons Namensnennung 4.0 International Lizenz veröffentlicht, welche die Nutzung, Vervielfältigung, Bearbeitung, Verbreitung und Wiedergabe in jegli-

\section{Ventral support in dorsal lumbar fusion surgery. A case report with many incidents}

Anterior column support in posterior lumbar fusion surgery is essential for the outcome of the operation, particularly in the case of unstable pathologies such as spondylolisthesis or spondylodiscitis. The complicated case of a patient with simultaneous spondylolisthesis and spondylodiscitis is presented and reevaluated based on the literature and our own treatment algorithm. In the case of spondylodiscitis alone, iliac crest interposal is sufficient as a support. In the case of additional disturbances of the sagittal profile, a cage implantation is preferred.

\section{Keywords}

Lumbar region · Spinal Fusion $\cdot$ Spine $\cdot$ Spondylodiscitis $\cdot$ Spondylolisthesis

chem Medium und Format erlaubt, sofern Sie den/die ursprünglichen Autor(en) und die Quelle ordnungsgemäßnennen, einen Link zur Creative Commons Lizenz beifügen und angeben, ob Änderungen vorgenommen wurden.

Die in diesem Artikel enthaltenen Bilder und sonstiges Drittmaterial unterliegen ebenfalls der genannten Creative Commons Lizenz, sofern sich aus der Abbildungslegende nichts anderes ergibt. Sofern das betreffende Material nicht unter der genannten Creative Commons Lizenz steht und die betreffende Handlung nicht nach gesetzlichen Vorschriften erlaubt ist, ist für die oben aufgeführten Weiterverwendungen des Materials die Einwilligung des jeweiligen Rechteinhabers einzuholen.

Weitere Details zur Lizenz entnehmen Sie bitte der Lizenzinformation auf http://creativecommons.org/ licenses/by/4.0/deed.de.

\section{Literatur}

1. Ames CP, Barry JJ, Keshavarzi S et al (2013) Perioperative outcomes and complications of pedicle subtraction osteotomy in cases with single versus two attending surgeons. Spine Deform 1:51-58. https://doi.org/10.1016/j.jspd.2012.10. 004

2. Ames CP, Smith JS, Scheer JK et al (2012) Impact of spinopelvic alignment on decision making in deformity surgery in adults: a review. J Neurosurg Spine 16:547-564. https://doi.org/10.3171/2012. 2.SPINE11320

3. Duarte RM, Vaccaro AR (2013) Spinal infection: state of the art and management algorithm. Eur Spine J 22:2787-2799. https://doi.org/10.1007/ s00586-013-2850-1

4. Ekman $P$, Moller $H$, Tullberg $T$ et al (2007) Posterior lumbar interbody fusion versus posterolateral fusion in adult isthmic spondylolisthesis. Spine 32:2178-2183. https://doi.org/10.1097/ BRS.0b013e31814b1bd8

5. Glassman SD, Bridwell K, Dimar JR et al (2005) The impact of positive sagittal balance in adult spinal deformity. Spine 30:2024-2029

6. Landham PR, Don AS, Robertson PA (2017) Do position and size matter? An analysis of cage and placement variables for optimum lordosis in PLIF reconstruction. Eur Spine J 26:2843-2850. https:// doi.org/10.1007/s00586-017-5170-z
7. Polly DW Jr., Klemme WR, Cunningham BW et al (2000) The biomechanical significance of anterior column support in a simulated single-level spinal fusion. JSpinal Disord 13:58-62

8. Robinson Y, Tschoeke SK, Finke T et al (2008) Successful treatment of spondylodiscitis using titanium cages: a 3-year follow-up of 22 consecutive patients. Acta Orthop 79:660-664. https://doi.org/ 10.1080/17453670810016687

9. Teng I, Han J, Phan K et al (2017) A meta-analysis comparing ALIF, PLIF, TLIF and LLIF. J Clin Neurosci 44:11-17. https://doi.org/10.1016/j.jocn.2017.06. 013 


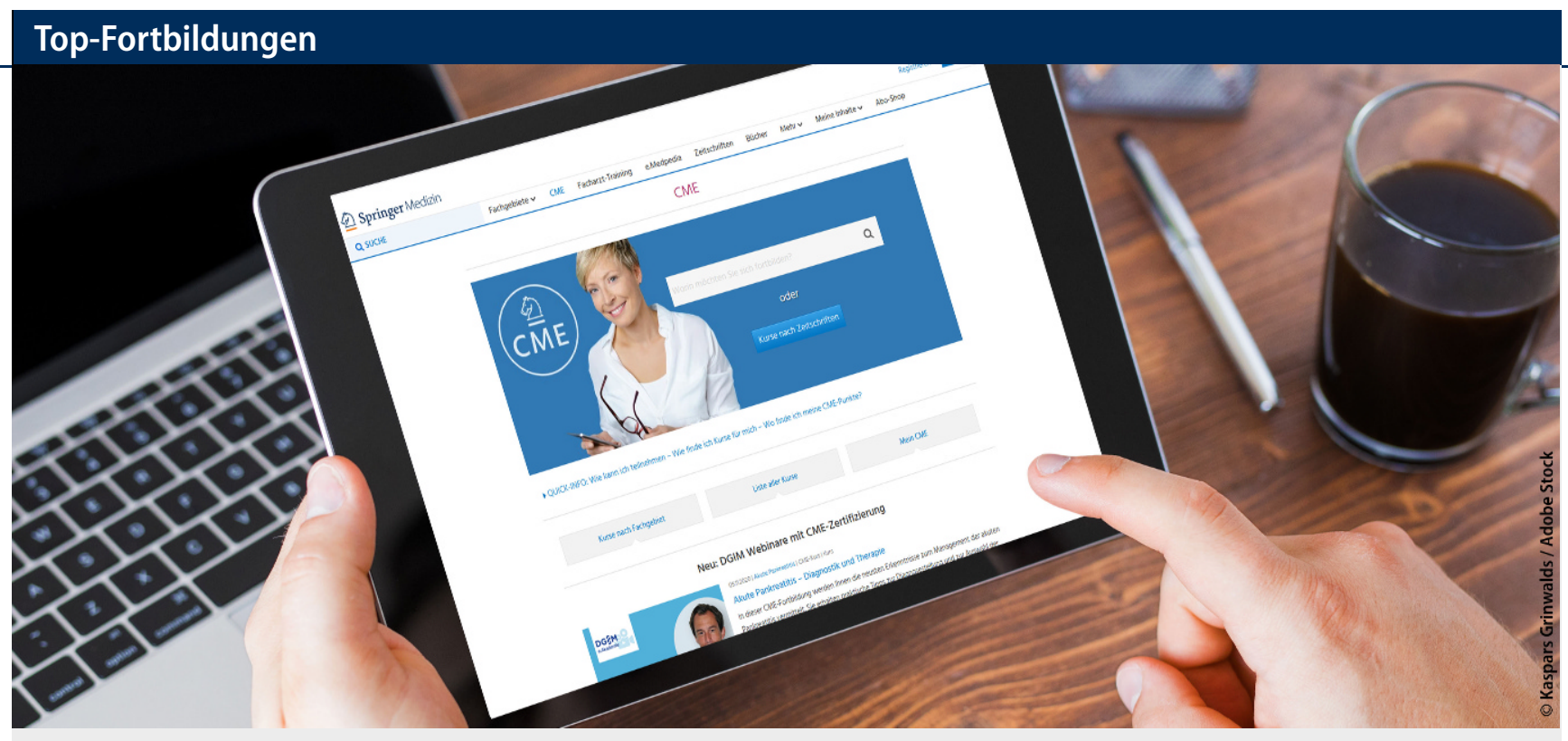

\section{Top-Fortbildungen Der Orthopäde}

Wir freuen uns sehr, Ihnen die von unseren Usern am besten bewerteten, aktuell noch zertifizierten CME-Kurse aus Der Orthopäde präsentieren zu dürfen. Jeder Teilnehmer hat nach der Bearbeitung eines Fortbildungsbeitrags auf Springermedizin.de die Möglichkeit, diesen zu bewerten und zu kommentieren. Im Namen der Schriftleitung und des Herausgeberboards von Der Orthopäde bedankt sich die Redaktion bei den Autorinnen und Autoren für die außerordentlich gut gelungenen Artikel. An allen aufgeführten CME-Kursen können Sie weiterhin teilnehmen, Sie finden sie unter https://www.springermedizin.de/derorthopaede.

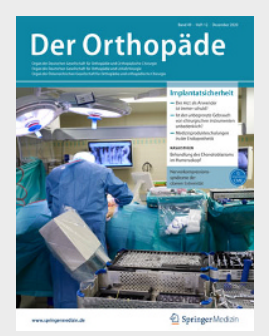

Nervenkompressionssyndrome der oberen Extremität aus: Der Orthopäde 12/2020 von: M. Zborilova, J. Grifka und L. Parik Userbewertung (Schulnote): 1,72 Zertifiziert bis: 01.12.2021

Zitat: „Sehr gut, sehr übersichtliche Darstellung der Diagnostik und Therapie"

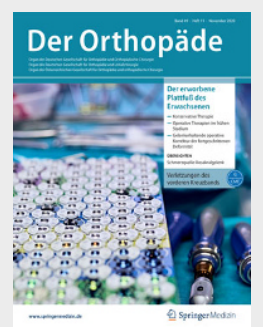

Verletzung des vorderen Kreuzbandes beim Erwachsenen aus: Der Orthopäde 11/2020 von: L. Kohn, E. Rembeck und A. Rauch Userbewertung (Schulnote): 1,72 Zertifiziert bis: 03.11.2021

Zitat: ,Sehr gute Übersicht zum gesamten Thema, v.a. zu den aktuellen differenzierten Therapiemöglichkeiten."

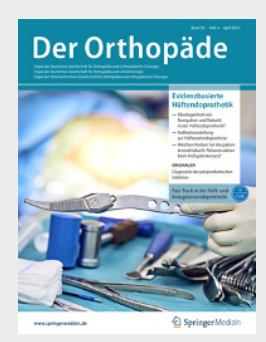

Fast-Track in der Hüft- und Kniegelenkendoprothetik

aus: Der Orthopäde 4/2021

von: F. Greimel, J. Grifka und G. Maderbacher Userbewertung (Schulnote): 1,74

Zertifiziert bis: 06.04.2022

Zitat: „Interssant und sehr gut strukturiert."

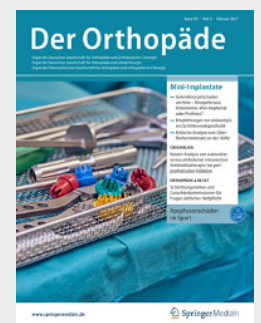

Apophysenschäden im Sport

aus: Der Orthopäde 2/2021

von: M. Neubauer und S. Nehrer

Userbewertung (Schulnote): 1,81

Zertifiziert bis: 16.02.2022

Zitat: „Interessanter Artikel, auch wegen der theoretischen Grundlagen." 